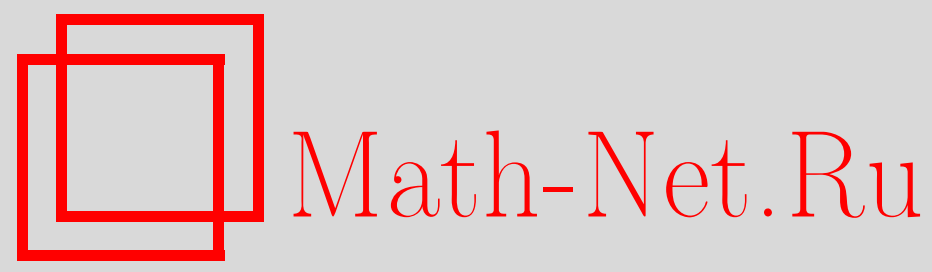

Р. Н. Бояринов, О больших расстояниях между последовательными нулями дзета-функции Римана, Дискрет. матем., 2010, том 22, выпуск 3, 75-82

DOI: https://doi.org/10.4213/dm1108

Использование Общероссийского математического портала Math-Net.Ru подразумевает, что вы прочитали и согласны с пользовательским соглашением http://www . mathnet.ru/rus/agreement

Параметры загрузки:

IP : 54.205 .225 .156

26 апреля 2023 г., $11: 52: 28$ 
УДК 511

\section{О больших расстояниях между \\ последовательными нулями дзета-функции Римана}

(ㄷ) 2010 г. .Н.Бояринов

Получена новая оценка числа нулей $\varrho_{n}=\beta_{n}+i \gamma_{n}$ дзета-функции Римана, ординаты $\gamma_{n}$ которой лежат на заданном интервале и для которых расстояние между соседними нулями является большим по сравнению со средним значением $2 \pi\left(\ln \left(\gamma_{n} /(2 \pi)\right)\right)^{-1}$.

\section{1. Введение}

Одним из основных направлений в теории дзета-функции Римана $\zeta(s)$ является изучение ее нулей, лежащих в критической полосе $0 \leqslant \operatorname{Re} s \leqslant 1$. Число $N(T)$ таких нулей, удовлетворяющих условию $0<\operatorname{Im} s \leqslant T$, выражается формулой Римана-Мангольдта (см. [1])

$$
N(T)=L(T)+S(T)+\frac{1}{\pi} \delta(T),
$$

где

$$
L(T)=\frac{T}{2 \pi} \ln \frac{T}{2 \pi}-\frac{T}{2 \pi}+\frac{7}{8},
$$

$S(T)$ - аргумент дзета-функции Римана, а $\delta(T)$ - гладкая функция, производная которой имеет оценку вида $\left|\delta^{\prime}(T)\right| \ll T^{-2}$.

Перенумеруем мнимые части нулей $\zeta(s)$ в критической полосе в порядке возрастания, а в случае совпадения нескольких ординат - в произвольном порядке:

$$
0<\gamma_{1}<\gamma_{2}<\ldots \leqslant \gamma_{n} \leqslant \gamma_{n+1} \leqslant \ldots
$$

Существует несколько утверждений, указывающих на то, что случаи, когда расстояние между последовательными ординатами велико, встречается достаточно редко.

Далее, если $\lambda \geqslant \lambda_{0}>0$, а целое число $r$ удовлетворяет условию

$$
1 \leqslant r \leqslant \lambda^{-1} T \ln T,
$$

то для числа $v_{r}$ пар $\gamma_{n}, \gamma_{n+r}$, удовлетворяющих условиям

$$
\frac{\gamma_{n+r}-\gamma_{n}}{r} \geqslant \frac{2 \pi \lambda}{\ln (T /(2 \pi))}, \quad T<\gamma_{n}, \quad \gamma_{n+r} \leqslant 2 T,
$$


А. Фуджи в работе [2] получил оценку

$$
v_{r} \leqslant c_{1} N(T) \exp \left(-c(\lambda r)^{2 / 3}(\ln \lambda r)^{-1 / 3}\right),
$$

где $c, c_{1}$ - положительные постоянные.

Далее, А. Ивич в работе [3] доказал, что число ординат $\gamma_{n}$, удовлетворяющих условиями

$$
T<\gamma_{n} \leqslant T+H, \quad H=T^{1 / 2+\varepsilon}, \quad \gamma_{n+1}-\gamma_{n} \geqslant \lambda(\ln T)^{-1}
$$

не превосходит $c_{1}(N(T+H)-N(T)) \exp (-c \lambda)$.

Одним из следствий теоремы А. Фуджи об оценке $v_{r}$ явилась верхняя оценка суммы

$$
S_{k}(T)=\sum_{T / 2<\gamma_{n} \leqslant T}\left(\gamma_{n+1}-\gamma_{n}\right)^{k}
$$

вида

$$
S_{k}(T) \leqslant c(k) \frac{N(T)}{(\ln T)^{k}}, \quad c(k)=\left(c_{1} k^{3 / 2} \ln (k+3)\right)^{k},
$$

где $k$ - целое число, $1 \leqslant k \leqslant c_{2}(T \ln T)^{2 / 3}$, а $c_{1}, c_{2}-$ некоторые абсолютные положительные постоянные.

В 1990 г. А. Фуджи в работе [4] улучшил свой результат при $k=2$, получив более точную оценку

$$
\sum_{T / 2<\gamma_{n} \leqslant T}\left(\gamma_{n+1}-\gamma_{n}\right)^{2} \leqslant 8,55 \cdot 2 \pi T / \ln \frac{T}{2 \pi}, \quad T>T_{0}>0 .
$$

Настоящая работа посвящена уточнению теорем, доказанных в [5]. Справедливы следующие утверждения.

Теорема 1. Пусть $\varepsilon-$ сколь угодно малое фиксированное число, $0<\varepsilon<0,001$, $T>T_{0}(\varepsilon)>0, H=T^{27 / 82+\varepsilon}$. Тогда для любого $\lambda \geqslant 4 \varkappa^{-1}$ для числа $v(\lambda ; T, H)$ ординат $\gamma_{n}$ нулей $\zeta(s)$, удовлетворяющих условиям

$$
\gamma_{n+1}-\gamma_{n} \geqslant \frac{2 \pi \lambda}{\ln (T /(2 \pi))}, \quad T<\gamma_{n}, \gamma_{n+1} \leqslant T+H,
$$

имеет место оиенка

$$
v(\lambda ; T, H)<\frac{e^{3}}{\lambda}(N(T+H)-N(T)) \exp (-\varkappa \lambda),
$$

где $\varkappa=(\pi / 3) e^{-19,5} \varepsilon^{1,5}$.

Замечание 1. Неравенство из теоремы 1 при $H=X^{\varepsilon}$ справедливо для всех $T$ из промежутка $(X, 2 X), X \geqslant X_{0}(\varepsilon)$, за исключением значений из некоторого множества, мера которого не превосходит $X^{1-0,01 \varepsilon}$.

Теорема 2. При любом $\lambda \geqslant 4 / \varkappa_{1} u T>T_{0}>0$ для числа $v(\lambda ; T)$ ординат $\gamma_{n}$ нулей $\zeta(s)$, удовлетворяющих условиям

$$
\gamma_{n+1}-\gamma_{n} \geqslant \frac{2 \pi \lambda}{\ln (T /(2 \pi))}, \quad T / 2<\gamma_{n} \leqslant T,
$$

имеет место оценка

$$
v(\lambda ; T)<\frac{e^{3}}{\lambda} N(T) \exp \left(-\varkappa_{1} \lambda\right), \quad \varkappa_{1}=\frac{9 \pi}{e^{19,5} 10^{6}} .
$$


Теорема 3. Пусть $T \geqslant T_{0}>0, k-$ произвольное положительное число, a $S_{k}(T)-$ сумма, определенная выме. Тогда имеет место оценка

$$
S_{k}(T) \leqslant \begin{cases}4,1 \varkappa_{2}^{k} N(T), & 0<k \leqslant 1, \\ \left(4^{k}+2,5 e^{4} \varkappa_{1} \Gamma(k)\right) \varkappa_{2}^{k} N(T), & k>1,\end{cases}
$$

где Г(·) - гамма-функичия и

$$
\varkappa_{1}=\frac{9 \pi}{e^{19,5} 10^{6}}, \quad \varkappa_{2}=\frac{2 \pi}{\varkappa_{1} \ln (T /(2 \pi))} .
$$

Теорема 4. Пусть $T \geqslant T_{0}>0, k-$ произвольное положительное число, а $S_{k}(T)-$ сумма, определенная выше. Тогда имеет место оценка

$$
S_{k}(T) \geqslant\left\{\begin{array}{l}
\frac{1}{34,24}\left(\frac{34,24 \pi}{\ln (T /(2 \pi))}\right)^{k} N(T), \quad 0<k<1, \\
4,1\left(\frac{\pi}{4,1 \ln (T /(2 \pi))}\right)^{k} N(T), \quad k \geqslant 1 .
\end{array}\right.
$$

\section{2. Вспомогательные леммы и доказательства теорем}

Лемма 1. Пусть $\varepsilon-$ сколь угодно малое фиксированное число, $0<\varepsilon<0,001$, $T>T_{0}(\varepsilon)>0, H=T^{27 / 82+\varepsilon}, x=T^{0,1 \varepsilon}, m-$ иелое число, $1 \leqslant m<(\ln x) / 192$, $x^{1 /(4 m)}<y \leqslant x^{1 / m}$. Тогда имеет место оценка

$$
\int_{T}^{T+H}\left(S(t)+\frac{1}{\pi} \sum_{p<y} \frac{\sin (t \ln p)}{\sqrt{p}}\right)^{2 m} d t<\left(e^{37} \pi^{-2} \varepsilon^{-3} m^{2}\right)^{m} H .
$$

Доказательство этого утверждения приведено на с. 51 в [6].

Лемма 2. Пусть $\varepsilon-$ сколь угодно малое фиксированное число, $0<\varepsilon<0,001$, $T>T_{0}(\varepsilon)>0$,

$$
0<\lambda<\frac{16 \ln (T /(2 \pi))}{\pi \ln \ln \ln T}, \quad H=T^{27 / 82+\varepsilon}, \quad h=\frac{\pi \lambda}{2 \ln (T /(2 \pi))},
$$

$m$ - иеелое число, $\left(\varepsilon^{5} \lambda^{2} /(9 B)\right)^{1 / 3} \leqslant m<(\ln x) / 192$. Тогда имеет место оценка

$$
I_{m}=\int_{T}^{T+H}(S(t+2 h)-S(t))^{2 m} d t<\left(9 B \varepsilon^{-3} m^{2}\right)^{m} H, \quad B=e^{37} \pi^{-2} .
$$

Доказательство. Положим $y=x^{1 / m}$ и рассмотрим тригонометрический полином

$$
U(t)=\sum_{p<y} a(p) p^{-0,5+i t}, \quad a(p)=p^{i h} \sin (h \ln p) .
$$

Тогда

$$
\begin{aligned}
S(t+2 h)-S(t)=(S(t+2 h)+ & \left.\frac{1}{\pi} \sum_{p<y} \frac{\sin ((t+2 h) \ln p)}{\sqrt{p}}\right) \\
& -\left(S(t)+\frac{1}{\pi} \sum_{p<y} \frac{\sin (t \ln p)}{\sqrt{p}}\right)-\frac{1}{\pi}(U(t)+\bar{U}(t)) .
\end{aligned}
$$


Возводя это выражение в степень $2 m$ и применяя к нему неравенство Гельдера, приходим к соотношению

$$
I_{m} \leqslant 3^{2 m-1}\left(2 J_{1}+J_{2}\right)
$$

в котором

$$
\begin{aligned}
& J_{1}=\int_{T}^{T+H+2 h}\left(S(t)+\frac{1}{\pi} \sum_{p<y} \frac{\sin (t \ln p)}{\sqrt{p}}\right)^{2 m} d t, \\
& J_{2}=\pi^{-2 m} \int_{T}^{T+H}(U(t)+\bar{U}(t))^{2 m} d t .
\end{aligned}
$$

Тогда из леммы 1 получаем оценку

$$
J_{1} \leqslant\left(B \varepsilon^{-3} m^{2}\right)^{m}(H+2 h) .
$$

Далее, интеграл $J_{2}$ представляется суммой

$$
J_{2}=\pi^{-2 m}\left(\left(\begin{array}{c}
2 m \\
m
\end{array}\right) H S_{m}+2 \theta \sum_{\nu=0}^{2 m}\left(\begin{array}{c}
2 m \\
v
\end{array}\right) \sum_{P_{\nu} \neq Q_{\nu}} \frac{1}{\sqrt{P_{\nu} Q_{\nu}}}\left|\ln \frac{P_{v}}{Q_{v}}\right|^{-1}\right),
$$

где

$$
S_{m}=\sum_{P_{m}=Q_{m}} \frac{\left|a\left(p_{1}\right)\right|^{2} \cdots\left|a\left(p_{m}\right)\right|^{2}}{\sqrt{P_{m} Q_{m}}},
$$

и через $P_{v}, Q_{v}$ обозначены произведения $p_{1} \cdots p_{v}$ и $q_{1} \cdots q_{2 m-v}$ соответственно. Так как наибольшее из чисел $P_{v}, Q_{v}$ при любом $v$ не превосходит $y^{2 m}$, а общее число пар $\left(P_{v}, Q_{v}\right)$ не превосходит $(\pi(y))^{2 m}$, верхней оценкой каждой из сумм по $P_{v} \neq Q_{v}$ служит величина

$$
(y \pi(y))^{2 m}<x^{4} \leqslant H^{0,2} .
$$

Поскольку число решений уравнения $p_{1} \cdots p_{m}=q_{1} \cdots q_{m}$ в простых числах $q_{1}, \ldots, q_{m}$ при фиксированной левой части не превосходит $m$ !, сумма $S_{m}$ удовлетворяет цепочке неравенств

$$
\begin{aligned}
S_{m} & \leqslant m !\left(\sum_{p<y} \frac{|a(p)|^{2}}{p}\right)^{m}=m !\left(\sum_{p<y} \frac{\sin ^{2}(h \ln p)}{p}\right)^{m} \leqslant m !\left(h^{2} \ln y \sum_{p<y} \frac{\ln p}{p}\right)^{m} \\
& \leqslant m !(2 h \ln y)^{2 m}=m !\left(\frac{\pi \lambda}{\ln (T /(2 \pi))} \frac{\varepsilon \ln T}{10 m}\right)^{2 m} \leqslant\left(\frac{\varepsilon \lambda}{3 \sqrt{m}}\right)^{2 m} .
\end{aligned}
$$

Следовательно,

$$
\begin{aligned}
J_{2} & \leqslant \pi^{-2 m}\left(\left(\begin{array}{c}
2 m \\
m
\end{array}\right) H S_{m}+2^{2 m+1} H^{0,2}\right) \leqslant \pi^{-2 m}\left(H\left(\frac{2 \varepsilon \lambda}{3 \sqrt{m}}\right)^{2 m}+2^{2 m} H^{0,3}\right) \\
& \leqslant 0,5 H\left(\frac{\varepsilon \lambda}{3 \sqrt{m}}\right)^{2 m}+0,1 H \leqslant 0,5\left(B \varepsilon^{-3} m^{2}\right)^{m} H+0,1 H \leqslant 0,6\left(B \varepsilon^{-3} m^{2}\right)^{m} H, \\
I_{m} & \leqslant 3^{2 m-1}\left(2\left(B \varepsilon^{-3} m^{2}\right)^{m}(H+2 h)+0,6\left(B \varepsilon^{-3} m^{2}\right)^{m} H\right) \leqslant\left(9 B \varepsilon^{-3} m^{2}\right)^{m} H .
\end{aligned}
$$

Лемма доказана. 
Доказательство теоремы 1. Из теоремы Литтлвуда [7] следует, что для $n>n_{0}$ верно неравенство $\gamma_{n+1}-\gamma_{n}<32\left(\ln \ln \ln \gamma_{n}\right)^{-1}<32(\ln \ln \ln T)^{-1}$. Поэтому можно считать, что

$$
\lambda<\frac{16 \ln (T /(2 \pi))}{\pi \ln \ln \ln T} \text {. }
$$

Положим

$$
h=\frac{\pi \lambda}{2 \ln (T /(2 \pi))} .
$$

Пусть для некоторой пары ординат $\gamma_{n}, \gamma_{n+1}$ из промежутка $(T, T+H]$ выполнено неравенство

$$
\gamma_{n+1}-\gamma_{n} \geqslant 4 h=\frac{2 \pi \lambda}{\ln (T /(2 \pi))} .
$$

Тогда при любом $t \in\left(\gamma_{n}, \gamma_{n}+2 h\right)$ справедливо равенство $N(t+2 h)-N(t)=0$, а соответствующие приращения $L(t)$ и $\delta(t)$ имеют вид

$$
\begin{aligned}
L(t+2 h)-L(t) & =\frac{h}{\pi} \ln \frac{t}{2 \pi}+\frac{h^{2}}{\pi(t+2 \theta h)}, \\
\delta(t+2 h)-\delta(t) & =\delta^{\prime}(t+2 \theta h) 2 h=O\left(h T^{-2}\right) .
\end{aligned}
$$

Следовательно,

$$
\begin{aligned}
& |S(t+2 h)-S(t)|=\left|L(t+2 h)-L(t)+\pi^{-1}(\delta(t+2 h)-\delta(t))\right| \\
& \quad=\frac{h}{\pi} \ln \frac{t}{2 \pi}+\frac{h^{2}}{\pi(t+2 \theta h)}+O\left(h T^{-2}\right) \geqslant \frac{h}{\pi} \ln \frac{T}{2 \pi}+\frac{h^{2}}{2 \pi(t+2 \theta h)}>\frac{h}{\pi} \ln \frac{T}{2 \pi}=\lambda / 2 .
\end{aligned}
$$

Отсюда получаем, что

$$
2 h(\lambda / 2)^{2 m} \leqslant \int_{\gamma_{n}}^{\gamma_{n}+2 h}(S(t+2 h)-S(t))^{2 m} d t \leqslant \int_{\gamma_{n}}^{\gamma_{n}+1}(S(t+2 h)-S(t))^{2 m} d t .
$$

Используя лемму 2 , получим, что для любого целого $m$, удовлетворяющего условию $\left(\varepsilon^{5} \lambda^{2} /(9 B)\right)^{1 / 3} \leqslant m<(\ln x) / 192$, справедливы неравенства

$$
2 h v(\lambda ; T, H)(\lambda / 2)^{2 m} \leqslant \int_{T}^{T+H}(S(t+2 h)-S(t))^{2 m} d t<\left(9 B \varepsilon^{-3} m^{2}\right)^{m} H, \quad B=e^{37} \pi^{-2} \text {. }
$$

Отсюда следует, что

$$
v(\lambda ; T, H) \leqslant H(2 h)^{-1}\left(\frac{3 e B^{0,5}}{\varepsilon^{1,5}} \frac{2 m}{e \lambda}\right)^{2 m}=H(2 h)^{-1}\left(\frac{2 m}{e \varkappa \lambda}\right)^{2 m} .
$$

Пусть $m=[0,5 \varkappa \lambda]$. Целое число $m=[0,5 \varkappa \lambda]$ удовлетворяет условиям

$$
m \leqslant 0,5 \varkappa 16 \ln T<10 \varkappa \ln T<\frac{0,1 \varepsilon \ln T}{192}=\frac{\ln x}{192}, \quad m>0,5 \varkappa \lambda-1 \geqslant 1 .
$$

Легко проверить, что при $\lambda \geqslant 4 \varkappa^{-1}$ также верно неравенство $m \geqslant\left(\varepsilon^{5} \lambda^{2} /(9 B)\right)^{1 / 3}$. Отсюда следует, что

$$
\begin{aligned}
\nu(\lambda ; T, H) & \leqslant H(2 h)^{-1} e^{-2 m}<e^{2} H(2 h)^{-1} \exp (-\varkappa \lambda)=\frac{H \ln (T /(2 \pi))}{2 \pi} \frac{2 e^{2}}{\lambda} \exp (-\varkappa \lambda) \\
& <\frac{2,2 e^{2}}{\lambda}(N(T+H)-N(T)) \exp (-\varkappa \lambda)<\frac{e^{3}}{\lambda}(N(T+H)-N(T)) \exp (-\varkappa \lambda) .
\end{aligned}
$$

Теорема доказана. 
Доказательство теоремы 2. Пусть $v(\lambda ; T, H)-$ функция из теоремы 1. Будем считать, что

$$
\lambda<\frac{16 \ln (T /(2 \pi))}{\pi \ln \ln \ln T},
$$

поскольку иначе (см. доказательство теоремы 1$)$ величина $v(\lambda ; T)$ тождественно равна нулю. Возьмем $\varepsilon=9 \cdot 10^{-4}$. Далее, определим числа $T_{s}, H_{s}, s=1,2, \ldots$, соотношениями $T_{1}=T, H_{s}=T_{s}^{27 / 82+\varepsilon}, T_{s+1}=T_{s}-H_{s}$ и положим $s_{0}$ равным номеру первого члена убывающей последовательности $T_{s}$ с условием $T_{s+1} \leqslant 0,5 T$. Очевидно, что $0,25 T<T_{s_{0}+1} \leqslant 0,5 T$ при достаточно большом $T$. Обозначим число ординат $\gamma_{n}$ нулей $\zeta(s)$, удовлетворяющих условиям

$$
\gamma_{n+1}-\gamma_{n} \geqslant \frac{2 \pi \lambda}{\ln (T /(2 \pi))}, \quad T_{s+1}<\gamma_{n}, \quad \gamma_{n+1} \leqslant T_{s},
$$

символом $\mu_{s}(\lambda ; T)$. Для любого $1 \leqslant s \leqslant s_{0}$ при достаточно большом $T$ верно неравенство

$$
\frac{2 \pi \lambda}{\ln (T /(2 \pi))} \geqslant \frac{2 \pi(\lambda-1)}{\ln \left(T_{s+1} /(2 \pi)\right)},
$$

поэтому

$$
\mu_{s}(\lambda ; T) \leqslant v\left(\lambda-1 ; T_{s+1}, H_{s}\right) .
$$

Согласно теореме 2 , для $1 \leqslant s \leqslant s_{0}$ при $\lambda \geqslant 4 / \varkappa_{1}$ справедливы неравенства

$$
\begin{aligned}
\mu_{s}(\lambda ; T) \leqslant v\left(\lambda-1 ; T_{s+1}, H_{s}\right) & \leqslant \frac{2,2 e^{2+\varkappa_{1}}}{\lambda-1}\left(N\left(T_{s}\right)-N\left(T_{s+1}\right)\right) \exp \left(-\varkappa_{1} \lambda\right) \\
& <\frac{2,5 e^{2}}{\lambda}\left(N\left(T_{s}\right)-N\left(T_{s+1}\right)\right) \exp \left(-\varkappa_{1} \lambda\right),
\end{aligned}
$$

где

сложив которые, получим, что

$$
\varkappa_{1}=\frac{\pi}{3} e^{-19,5} \varepsilon^{1,5}=\frac{9 \pi}{e^{19,5} \cdot 10^{6}},
$$

$$
v(\lambda ; T) \leqslant \sum_{s \leqslant s_{0}} \mu_{s}(\lambda ; T) \leqslant \frac{2,5 e^{2}}{\lambda}\left(N\left(T_{1}\right)-N\left(T_{s_{0}+1}\right)\right) \exp \left(-\varkappa_{1} \lambda\right)<\frac{e^{3}}{\lambda} N(T) \exp \left(-\varkappa_{1} \lambda\right) .
$$

Теорема доказана.

Доказательство теоремь 3. Все ординаты $\gamma_{n} \in(0,5 T, T]$ распределим по классам $E_{4}, E_{5}, E_{6}, \ldots, E_{r}, \ldots$, относя к классу $E_{r}$ при $r \geqslant 5$ те из них, которые удовлетворяют условию

$$
\frac{2 \pi(r-1)}{\varkappa_{1} \ln (T /(2 \pi))} \leqslant \gamma_{n+1}-\gamma_{n}<\frac{2 \pi r}{\varkappa_{1} \ln (T /(2 \pi))} .
$$

В класс $E_{4}$ отнесем те $\gamma_{n} \in(0,5 T, T]$, которые удовлетворяют условию

$$
\gamma_{n+1}-\gamma_{n}<\frac{8 \pi}{\varkappa_{1} \ln (T /(2 \pi))}
$$

В силу теоремы 2, число элементов в $E_{r}$ при $r \geqslant 5$ не превосходит

$$
\frac{\varkappa_{1} e^{4}}{r-1} N(T) e^{-r}=\frac{\varkappa_{1} e^{4}}{r} N(T) e^{-r} \frac{r}{r-1} \leqslant \frac{5 \varkappa_{1} e^{4}}{4 r} N(T) e^{-r},
$$


а в $E_{4}$ не превосходит $N(T)$. Поэтому для любого $k>0$ верно неравенство

$$
S_{k}(T) \leqslant 4^{k}\left(\frac{2 \pi}{\varkappa_{1} \ln (T /(2 \pi))}\right)^{k} N(T)+\frac{5 \varkappa_{1} e^{4}}{4}\left(\frac{2 \pi}{\varkappa_{1} \ln (T /(2 \pi))}\right)^{k} N(T) \sum_{r=5}^{+\infty} r^{k-1} e^{-r} .
$$

Заметим, что $S_{1}(T)=0,5 T+o(1)$. Разберем случай $0<k \leqslant 1$. Тогда

$$
\sum_{r=5}^{\infty} r^{k-1} e^{-r} \leqslant \sum_{r=5}^{\infty} e^{-r}=\frac{e^{-4}}{e-1}<\frac{2}{3} e^{-4}
$$

Отсюда следует, что

$$
S_{k}(T) \leqslant\left(4^{k}+5 \varkappa_{1} / 6\right)\left(\frac{2 \pi}{\varkappa_{1} \ln (T /(2 \pi))}\right)^{k} N(T) \leqslant 4,1\left(\frac{2 \pi}{\varkappa_{1} \ln (T /(2 \pi))}\right)^{k} N(T) .
$$

Рассмотрим теперь случай $k>1$. Заметим, что функция $f(x)=x^{k-1} e^{-x}$ убывает на промежутке $(0, k-1)$, возрастает на $(k-1, \infty)$, а в точке $x=k-1$ достигает своего максимума. Если $k \in \mathbf{N}$, то

$$
\sum_{r=5}^{\infty} f(r)<\int_{0}^{\infty} f(x) d x+((k-1) / e)^{k-1}<2 \Gamma(k) .
$$

Если $k \notin \mathbf{N}$, то

$$
\sum_{r=5}^{\infty} f(r)<\int_{0}^{+\infty} f(x) d x=\Gamma(k)
$$

Отсюда получаем, что

$$
S_{k}(T) \leqslant\left(4^{k}+2,5 \varkappa_{1} e^{4} \Gamma(k)\right)\left(\frac{2 \pi}{\varkappa_{1} \ln (T /(2 \pi))}\right)^{k} N(T) .
$$

Теорема доказана.

Доказательство теоремь 4. Рассмотрим случай $0<k<1$. Воспользуемся доказанным в [4] неравенством

$$
\sum_{0,5 T<\gamma_{n} \leqslant T}\left(\gamma_{n+1}-\gamma_{n}\right)^{2} \leqslant 8,55 \frac{2 \pi T}{\ln (T /(2 \pi))}, \quad T>T_{0}>0 .
$$

Отсюда следует, что

$$
\sum_{0,5 T<\gamma_{n} \leqslant T}\left(\gamma_{n+1}-\gamma_{n}\right)^{2}<8,56\left(\frac{2 \pi}{\ln (T /(2 \pi))}\right)^{2} N(T), \quad T>T_{0}>0 .
$$

Применяя неравенство Гельдера к сумме $\sum_{0,5 T<\gamma_{n} \leqslant T}\left(\gamma_{n+1}-\gamma_{n}\right)>0,5 T$, получаем неравенство

$$
\begin{aligned}
\frac{\pi N(T)}{\ln (T /(2 \pi))} & <\sum_{0,5 T<\gamma_{n} \leqslant T}\left(\gamma_{n+1}-\gamma_{n}\right) \\
& \leqslant\left(\sum_{0,5 T<\gamma_{n} \leqslant T}\left(\gamma_{n+1}-\gamma_{n}\right)^{k}\right)^{1 /(2-k)}\left(\sum_{0,5 T<\gamma_{n} \leqslant T}\left(\gamma_{n+1}-\gamma_{n}\right)^{2}\right)^{(1-k) /(2-k)} .
\end{aligned}
$$


Отсюда получаем оценку снизу для $S_{k}(T)$

$$
\sum_{0,5 T<\gamma_{n} \leqslant T}\left(\gamma_{n+1}-\gamma_{n}\right)^{k} \geqslant \frac{1}{34,24}\left(\frac{34,24 \pi}{\ln (T /(2 \pi))}\right)^{k} N(T) .
$$

Пусть теперь $k>1$. Снова применим неравенство Гельдера к $\sum_{0,5 T<\gamma_{n} \leqslant T}\left(\gamma_{n+1}-\gamma_{n}\right)$. Пусть $0<\alpha<1 / k$, тогда верно неравенство

$$
\begin{aligned}
\frac{\pi N(T)}{\ln (T /(2 \pi))} & <\sum_{0,5 T<\gamma_{n} \leqslant T}\left(\gamma_{n+1}-\gamma_{n}\right) \\
& \leqslant\left(\sum_{0,5 T<\gamma_{n} \leqslant T}\left(\gamma_{n+1}-\gamma_{n}\right)^{k}\right)^{\alpha}\left(\sum_{0,5 T<\gamma_{n} \leqslant T}\left(\gamma_{n+1}-\gamma_{n}\right)^{(1-\alpha k) /(1-\alpha)}\right)^{1-\alpha} .
\end{aligned}
$$

Отсюда получаем оценку снизу для $S_{k}(T)$

$$
\sum_{0,5 T<\gamma_{n} \leqslant T}\left(\gamma_{n+1}-\gamma_{n}\right)^{k} \geqslant 4,1\left(\frac{\varkappa_{1}}{8,2}\right)^{1 / \alpha}\left(\frac{2 \pi}{\varkappa_{1} \ln (T /(2 \pi))}\right)^{k} N(T) .
$$

Перейдем в этом неравенстве к пределу при $\alpha \rightarrow 1 / k-0$, тогда получим, что

$$
\sum_{0,5 T<\gamma_{n} \leqslant T}\left(\gamma_{n+1}-\gamma_{n}\right)^{k} \geqslant 4,1\left(\frac{\pi}{4,1 \ln (T /(2 \pi))}\right)^{k} N(T) .
$$

Заметим, что это неравенство верно и при $k=1$. Теорема доказана.

\section{Список литературы}

1. Карацуба А. А., Королев М. А., Аргумент дзета-функции Римана. Успехи матем. наук (2005) 60 , №3 (363), 41-96.

2. Fujii A., On the difference between $r$ consecutive ordinates of the zeros of the Riemann zeta-function. Proc. Japan Acad. (1975) 51, 741-743.

3. Ivić A., On small values of the Riemann zeta-function on the critical line and gaps between zeros. Liet. Mat. Rink. (2002) 42, 25-36.

4. Fujii A., On the gaps between consecutive zeros of the Riemann zeta-function. Proc. Japan Acad., Ser. Math. Sci. (1990) 66, 97-100.

5. Королев М. А., О больших расстояниях между соседними нулями дзета-функции Римана. Изв. РАН, сер. матем. (2008) 72, №2, 91-104.

6. Карацуба А. А., Королев М. А., Поведение аргумента дзета-функции Римана на критической прямой. Успехи матем. наук (2006) 61, №3 (369), 3-92.

7. Littlewood J. E., On the zeros of the Riemann zeta-function. Proc. Cambridge Phil. Soc. (1924) 22, 295-318. 